\title{
Dopamine dysregulation syndrome in Parkinson's disease: from clinical and neuropsychological characterisation to management and long-term outcome
}

\author{
Roberto Cilia, Chiara Siri, Margherita Canesi, Anna Lena Zecchinelli, \\ Danilo De Gaspari, Francesca Natuzzi, Silvana Tesei, Nicoletta Meucci, \\ Claudio Bruno Mariani, Giorgio Sacilotto, Michela Zini, Claudio Ruffmann, \\ Gianni Pezzoli
}

- Additional material is published online only. To view please visit the journal online (http://dx.doi.org/10.1136/ jnnp-2012-303988)

Parkinson Institute, Istituti Clinici di Perfezionamento, Milan, Italy

\section{Correspondence to} Dr Roberto Cilia, Parkinson Institute, Istituti Clinici di Perfezionamento, via Bignami 1, Milan 20126, Italy;

roberto.cilia@gmail.com roberto.cilia@icp.mi.it

Received 22 August 2012 Revised 29 January 2013 Accepted 11 March 2013 Published Online First 16 April 2013

\section{SLinked}

- http://dx.doi.org/10.1136/ jnnp-2013-305378

\footnotetext{
To cite: Cilia $R$, Siri $C_{\text {, }}$ Canesi M, et al. J Neurol Neurosurg Psychiatry 2014;85:311-318.
}

\begin{abstract}
Objective Dopamine dysregulation syndrome (DDS) refers to a compulsive pattern of dopaminergic drug misuse complicating Parkinson's disease (PD). To date, few data are available on DDS risk factors, cognitive profile and long-term outcome.

Methods In this retrospective case-control study, consecutive PD outpatients fulfilling criteria for DDS were assessed over a 6-year period (2005-2011). They were compared with 70 PD cases matched for age at onset, gender and disease duration, and with 1281 subjects with motor fluctuations and dyskinesias. DDS patients and matched controls underwent extensive neuropsychological assessment. Strategies for DDS patients management and the outcome at the last follow-up visit were recorded.
\end{abstract}

Results Thirty-five patients with DDS were identified, reporting history of depression, family history of PD and drug abuse, greater difference between 'Off' versus 'On' motor symptoms compared to age-matched controls. They had younger age at onset (but not any gender difference) compared to general PD population. Cognitive profile of DDS did not show major abnormalities, including executive functions. DDS patients have been followed up for 3.2 2 2.1 years and remission was recorded in $40 \%$ of cases. Negative DDS outcome was significantly associated with poor caregiver supervision. Sustained remission occurred more commonly on clozapine and on duodenal levodopa infusion and subthalamic nucleus deep brain stimulation (STN-DBS) than on apomorphine pump treatment.

Conclusions Clinicians should be aware of risk factors predisposing to DDS. Duodenal levodopa infusion and, less consistently, STN-DBS were more commonly associated with DDS remission. Effective caregiving plays a key role in long-term behavioural outcome.

\section{INTRODUCTION}

Parkinson's disease (PD) is a neuropsychiatric disorder characterised by motor and non-motor symptoms. The former result from nigrostriatal dopaminergic neuronal loss and the latter include dysfunction within a neural system involved in reward signalling, which shape goal-directed behaviours, that is, the mesocortical and mesolimbic system. Dopamine replacement therapy (DRT), used to restore normal dopamine levels in motor pathways and relieve motor symptoms, may stimulate the mesocorticolimbic system abnormally in a minority of vulnerable cases leading to addictive disorders, such as impulse control disorders (ICDs) and compulsive dopaminergic drug misuse (also called 'hedonistic homeostatic dysregulation' or 'dopamine dysregulation syndrome', DDS). ${ }^{1}{ }^{2}$ DDS is a non-motor complication of DRT occurring in $3-4 \%$ of patients with advanced PD, who develop an addictive pattern of medication intake, characterised by the intake of large doses of dopaminergic drugs in excess of that required to control motor symptoms, continual requests to the physician for larger doses of DRT or self-escalation of these medications without medical approval despite severe social destructive behaviours. ${ }^{1}$

Although the first reports date back about 30 years, ${ }^{3} 4$ the awareness of a pattern of compulsive dopaminergic medication intake possibly complicating a minority of PD patients increased rapidly in the early 2000s. ${ }^{1}{ }^{5-7}$ In recent years, however, relatively poor attention has been given to DDS compared to ICDs despite its highly distressing personal and familial negative consequences. There are several features of DDS that still need to be elucidated ${ }^{8}$ to help clinicians to allow preventive strategies, early detection and management, and improve the understanding of the underlying mechanisms in order to limit potential harm.

In the present study, we report clinical and neuropsychological (NPS) features of a large sample of PD patients with DDS and provide some learning lessons about the outcome of this uncommon addictive disorder, depending on the management proposed to the patients followed during a 6-year period.

\section{METHODS}

In this retrospective longitudinal populationbased cohort study, consecutive outpatients fulfilling UK Brain Bank criteria for $\mathrm{PD}^{9}$ and attending the Parkinson Institute (Istituti Clinici di Perfezionamento, Milan, Italy) from April 2005 to April 2011 were assessed for the presence of 
compulsive dopaminergic drug intake and followed by the same neurologist over time. During routine neurological examination, patients and their caregivers were asked to provide a list of all current medications and their dosages and were interviewed to ascertain compliance to treatment. If any behavioural/psychiatric issue was suspected, patients underwent full NPS assessment by experienced neuropsychologists (CS, DDG) to confirm the diagnosis of DDS according to working diagnostic criteria. ${ }^{1}$ All historical and follow-up data were collected from our electronic clinical database (a flow chart is provided as an online supplementary figure). According to clinical practice requirements, each neurologist scheduled follow-up visits every 13 months and recorded DDS persistence versus remission according to Giovannoni criteria (still vs no-longer fulfilling B-to-E criteria, ${ }^{1}$ respectively). We excluded patients with less than 6-month neurological follow-up, those with 'Off'-related painful dystonia, ${ }^{1}$ those with suspected DDS at first assessment who did not undergo NPS evaluation, and those who developed DDS after the initiation of subthalamic nucleus deep brain stimulation (STN-DBS), subcutaneous continuous apomorphine infusion (CAI), or duodenal levodopa infusion (DLI). Historical account included occupation, family history of $\mathrm{PD}$, present/past cigarette smoking, personal/immediate family history of experimental drug use, and history of depression $>5$ years prior to PD onset. Genetic counselling was performed in all those reporting positive family history of PD. Effective versus poor control by family members/caregiver over compliance to treatment was recorded.

Clinical assessment included the complete Unified Parkinson Disease Rating Scale (UPDRS) from part I to part IV. UPDRS part II scores (activities of daily living), UPDRS part III scores (motor examination) and the Hoehn and Yahr stage were rated according to patients' daily 'worst-Off' and 'best-On' condition. Motor fluctuations were assessed using the UPDRS part IV scores: dyskinesias/dystonia (sum of items 32-to-35) and 'Off' periods (sum of items 36-to-39). Body mass index (BMI, calculated as: weight/height ${ }^{2}$ ) and levodopa daily dosage/BMI ratio were also recorded. ${ }^{10}$

As control subjects, we used a group of PD patients matched by gender, education, age at onset, and disease duration consecutively recruited during the 2010-2011 period in a 1:2 case-to-control ratio, whose adherence to treatment was confirmed by caregivers (table 1A). We additionally compared demographic and clinical features of DDS subjects with those of

Table 1 Clinical features of PD patients with DDS vs matched control subjects at baseline

\begin{tabular}{|c|c|c|c|}
\hline & $\operatorname{DDS}(n=35)$ & Controls $(n=70)$ & $\mathrm{p}$ Values \\
\hline \multicolumn{4}{|l|}{ (A) Demographic features } \\
\hline Age at PD onset, years & 46.5 (11.6) & $47.5(9.2)$ & 0.6 \\
\hline Disease duration, years & $15.1(4.9)$ & $14.1(4.1)$ & 0.29 \\
\hline Male gender, \% & 68 & 62 & 0.53 \\
\hline Family history of PD in first degree relatives ( $\mathrm{n}(\%)$ ) & $16(46)$ & $15(21)$ & 0.009 \\
\hline Depression prior to PD onset ( $\mathrm{n} \%)$ & $19(54)$ & $21(30)$ & 0.017 \\
\hline Impulse Control Disorders (n (\%)) & $23(66)$ & $9(13)$ & $<0.001$ \\
\hline Smokers (n (\%)) & $14(40)$ & $23(33)$ & 0.53 \\
\hline Personal or family history of drug use ( $\mathrm{n}(\%))$ & $6(19)$ & $2(3)$ & 0.012 \\
\hline \multicolumn{4}{|l|}{ (B) UPDRS scores and Motor features } \\
\hline UPDRS part I-item 2 (Thought disorder) & $1.76(1.2)$ & $0.5(0.7)$ & $<0.001$ \\
\hline UPDRS part II—Off & $19.6(6.8)$ & $18.8(5.9)$ & 0.57 \\
\hline UPDRS part III—Off & $42.3(13.7)$ & $34.1(8.6)$ & 0.001 \\
\hline$\Delta$ UPDRS part III 'Off'-'On' & $25.3(9.6)$ & $18.5(7.5)$ & $<0.001$ \\
\hline UPDRS part IV—Dyskinesias (items 32 to 35 ) & $4.6(1.5)$ & $3.7(1.4)$ & 0.003 \\
\hline UPDRS part IV—Off (items 36 to 39) & $2.7(0.8)$ & $2.6(0.9)$ & 0.42 \\
\hline Hoehn and Yahr stage-Off & $3.2(0.7)$ & $3.2(0.8)$ & 0.85 \\
\hline Disabling dyskinesias* (n (\%)) & $30(86)$ & $32(46)$ & 0.006 \\
\hline \multicolumn{4}{|l|}{ (C) Therapy features } \\
\hline Total LEDD, (mg/day) & $\begin{array}{l}2327(1570) \\
{[1000-7200]}\end{array}$ & $\begin{array}{l}1107(436) \\
{[600-2600]}\end{array}$ & $<0.001$ \\
\hline Levodopa dosage, (mg/day) & $\begin{array}{c}1486(956) \\
{[0-6000]}\end{array}$ & $\begin{array}{l}751(390) \\
{[350-2250]}\end{array}$ & $<0.001$ \\
\hline Daily levodopa doses (n (SD) [range]) & $\begin{array}{c}8.4(3.6) \\
{[0-18]}\end{array}$ & $\begin{array}{c}6.3(1.4) \\
{[4-12]}\end{array}$ & $<0.001$ \\
\hline Disease duration at levodopa initiation, years & $\begin{array}{c}3.0(3.3) \\
{[0-18]}\end{array}$ & $\begin{array}{c}3.2(2.8) \\
{[0-13]}\end{array}$ & 0.79 \\
\hline Dopamine agonist dosage, (mg/day) & $\begin{array}{c}845(1245) \\
{[0-5200]}\end{array}$ & $\begin{array}{r}357(143) \\
{[50-650]}\end{array}$ & $<0.001$ \\
\hline Dopamine agonist (\% total LEDD, (SD)) & $30.5(25)$ & $32.3(10.4)$ & 0.56 \\
\hline Antidepressant therapy (n (\%)) & $11(31)$ & $9(13)$ & 0.035 \\
\hline Antipsychotic therapy (n (\%)) & $23(66)$ & $11(16)$ & $<0.001$ \\
\hline Advanced-stage treatment $(\mathrm{n}(\%)) \dagger$ & $17(49)$ & $22(31)$ & 0.20 \\
\hline
\end{tabular}

Values are given as 'mean (SD) [range]' or 'n/total (\%)'.

*Disabling dyskinesias has been defined as UPDRS IV (items $32+33$ ) $\geq 4$.

†Advanced stage treatment include: continuous apomorphine infusion, duodenal levodopa infusion and subthalamic nucleus deep brain stimulation. DDS, dopamine dysregulation syndrome; LEDD, levodopa-equivalent daily dose; PD, Parkinson's disease; UPDRS, Unified Parkinson's Disease Rating Scale. 
1281 sequential unrelated PD patients with motor fluctuations and dyskinesias whom we considered to be representative of the general population with advanced PD and motor complications. These data have been retrieved from the Parkinson Institute clinical and research database according to the following criteria: sum of UPDRS part IV items 32-to-35 for dyskinesias and the sum of items 36 -to-39 for 'Off' period scores $\neq 0$ in at least two neurological examinations performed $>6$ months apart (see online supplementary table). All PD patients with DDS and matched PD controls underwent extensive NPS assessment at the baseline, preceded by a $30 \mathrm{~min}$ semi-structured interview aiming to confirm the presence of pathological behaviours according to current diagnostic criteria for DDS ${ }^{1}$ and ICDs. ${ }^{11}$ Patients and caregivers were independently interviewed. DDS patients were assessed within 6 months from the onset of addictive symptoms. NPS testing included assessment of general cognition (mini-mental state examination, $\mathrm{MMSE}^{12}$ ), executive functions (the frontal assessment battery, $\mathrm{FAB}^{13}$ ), phonological and semantic verbal fluencies, ${ }^{14}$ Raven's coloured progressive matrices, ${ }^{15}$ selective and sustained attention (attentive matrices ${ }^{16}$ ), and memory functions (the Corsi block tapping test ${ }^{17}$ ), the digit span test, ${ }^{18}$ the Rey auditory verbal learning test. ${ }^{19}$ We used the 30 -items geriatric depression scale $\left(\mathrm{GDS}-30^{20}\right)$ to assess depressive symptoms and the neuropsychiatric inventory (NPI) to investigate psychiatric disturbances (this is a questionnaire where information on the patient's behaviour is obtained from the caregiver ${ }^{21}$ ). Testing was performed in the morning on their usual medication and scores were adjusted for age and education when appropriate; dichotomous variables ('normal' vs 'pathologic') were additionally calculated according to normative cut-off values. Individual daily medication dose was calculated as the sum of the levodopa and dopamine agonists converted into levodopa-equivalent daily dose (LEDD). ${ }^{22} 23$

Informed consent was obtained according to the Declaration of Helsinki. The study was approved by the ethics committee of our institution.

\section{Statistical analysis}

Data were analysed using the software program SPSS (Windows Release 17.0; SPSS Inc, Chicago, Illinois, USA). To compare DDS patients and control groups, parametric methods were used for variables after testing for normal distribution (Shapiro-Wilk W statistic). Comparisons between continuous variables were performed using the Mann-Whitney $U$ test or the Student $\mathrm{t}$ test as appropriate, according to normal distribution of data. Differences in categorical variables were assessed by means of the $\chi^{2}$ test or Fisher's exact test, as appropriate. Wilcoxon test was used for paired comparisons. We used a binary logistic forward LR regression model to investigate those clinical factors (independent variables) that predict the diagnosis of DDS (dependent variable), after assessing collinearity between variables through Pearson's statistics. ORs and 95\% CIs are reported for logistic regression models. The Hosmer-Lemeshow goodness-of-fit test was used to evaluate how well the multivariable models fit the data.

\section{RESULTS}

Over the 6-year study period, forty-seven PD patients were suspected of compulsive abuse of dopaminergic medication. According to a priori, 12 of these subjects $(7 \mathrm{M} / 5 \mathrm{~F}$, age at PD onset $46.8 \pm 8.5$ ) were excluded because: they did not perform NPS assessment and/or were lost before the 6-month follow-up visit $(\mathrm{n}=8 ; 5 \mathrm{M} / 3 \mathrm{~F}$, age at $\mathrm{PD}$ onset $46.1 \pm 9.7$; disease duration 14.5 (5.3) years; mean LEDD 2326 (980) mg/day) or because self-medication aimed at preventing painful 'Off'-related dystonia ( $\mathrm{n}=4$; all of them did not fulfil complete $\mathrm{DDS}^{1}$ and all remitted soon after medical therapy optimisation (also including anticholinergics and resolution of painful dystonic symptoms). In our cohort, we did not find anyone who developed DDS after DBS or any infusion treatment.

\section{DDS features}

\section{Demographic features}

Mean clinical follow-up of PD patients with DDS was approximately 3 years, ranging from 6 months to 6 years. A pattern of compulsive intake of levodopa was present in 32 subjects (91\%), while three patients had a severe addiction to dopamine agonists (9\%; $n=2$ pramipexole; $n=1$ cabergoline), either before or after levodopa initiation. About half of DDS cases had a family history of PD, more than twice the frequency in agematched controls. When compared to sequential subjects with advanced PD (Control B in see online supplementary table), patients with DDS were younger by approximately 10 years at onset. History of depression was significantly higher in the DDS group, but depressive symptoms and family history of PD were not correlated to each other). Personal and/or immediate family history of substance abuse was recorded in six DDS patients (table 1). Four DDS patients had personal history of substance abuse $(3 \mathrm{M} / 1 \mathrm{~F}$; alcohol $\mathrm{n}=2$; cocaine $\mathrm{n}=1$; alcohol+cocaine theroin + marijuana $n=1)$ ), one of whom with family history as well (father with alcohol abuse). Severe nicotine addiction (>40 cigarette/day) was recorded in two more male DDS cases. ${ }^{2}$ Moreover, two female DDS patients had an immediate family (but not personal) history of substance abuse: one whose son had multiple drug addictions and another whose brother had PD and history of cocaine abuse.

\section{Motor features and therapy at baseline}

DDS patients displayed more severe motor fluctuations compared to matched control subjects, with greater difference of individual UPDRS motor scores between 'Off' and 'On' condition ( $\triangle$ UPDRS in table $1 \mathrm{~B}$ ), worse UPDRS part III scores in the 'Off' (but not in the 'On') state and higher UPDRS-IV subscores for involuntary movements (but not those related to daily 'Off'). Compulsive medication intake induced patients to take significantly higher doses of either levodopa (up to $2250 \mathrm{mg}$ / day) and/or dopamine agonists (up to 3650 LEDD) than matched controls. The number of daily doses of levodopa (and of pramipexole in one case) was as high as 18-20 times/day in some cases.

\section{NPS and psychiatric features}

Overall, patients with DDS and matched PD controls had a similar cognitive profile, with the exception of worse performance at attentive matrices in the former group (table 2). Neuropsychiatric profile of DDS patients revealed worse depressive symptoms (according to GDS-30 and NPI), disinhibition, irritability, and delusions (but not hallucinations). The latter finding in DDS is consistent with the higher scores of the 'thought disorders' item of UPDRS part I (table 1). Psychosis and depression were not correlated to each other. Impulse control disorders were more common among DDS patients: at least one impulse control disorder was diagnosed in two-thirds of DDS cases, seven of whom had multiple ICDs. A NPS follow-up assessment (T1) was available only in 5/35 (14\%) of DDS patients $(4 \mathrm{M} / 1 \mathrm{~F} ; \mathrm{n}=3 / 5$ non-remitters). After a similar 2-year follow-up from the baseline assessment (T0), remitters $(1 \mathrm{M} / 1 \mathrm{~F})$ showed an improvement in main cognitive and 
Table 2 Neuropsychological assessment in DDS and matched PD control subjects

\begin{tabular}{|c|c|c|c|c|c|c|}
\hline \multirow[b]{2}{*}{ Test } & \multicolumn{3}{|c|}{ Mean (SD) scores } & \multicolumn{3}{|c|}{ Dichotomous values (\% pathological) } \\
\hline & DDS & Controls & p Values & DDS & Controls & p Values \\
\hline MMSE & $28.1(1.8)$ & $28.6(1.2)$ & 0.085 & 8 & 0 & 0.079 \\
\hline FAB & $13.6(3.2)$ & $14.8(2.6)$ & 0.092 & 41 & 25 & 0.261 \\
\hline CPM_Raven & $28.6(4.5)$ & $28.8(4.7)$ & 0.877 & 5 & 4 & 0.991 \\
\hline Phonological fluency & $32.3(11.7)$ & $30.1(10.9)$ & 0.455 & 0 & 8 & 0.303 \\
\hline Semantic fluency & $38.1(10.1)$ & $38.0(8.2)$ & 0.991 & 11 & 4 & 0.193 \\
\hline RAVLT learning & $36.1(9.9)$ & $39.8(9.9)$ & 0.180 & 21 & 16 & 0.721 \\
\hline RAVLT recall & $7.8(2.8)$ & $8.5(3.2)$ & 0.366 & 16 & 9 & 0.422 \\
\hline Denomination & $17.7(2.1)$ & $18.2(1.9)$ & 0.420 & 7 & 2 & 0.441 \\
\hline Digit span & $5.4(1.1)$ & $5.5(1.0)$ & 0.847 & 0 & 0 & 1.000 \\
\hline Digit back & $3.6(1.0)$ & $4.1(1.2)$ & 0.091 & NA & NA & NA \\
\hline Corsi block tapping test & $4.1(1.1)$ & $4.4(1.1)$ & 0.329 & 21 & 14 & 0.479 \\
\hline Attentive matrices & $39.1(11.2)$ & $46.8(10.9)$ & 0.032 & 22 & 4 & 0.021 \\
\hline GDS-30 & $14.2(5.9)$ & $10.2(6.6)$ & 0.045 & 61 & 27 & 0.044 \\
\hline NPI total & $38.2(23.2)$ & $24.4(17.9)$ & 0.008 & NA & NA & NA \\
\hline NPI 1/12: delusions & $3.0(5.1)$ & $0.7(2.1)$ & 0.046 & - & - & - \\
\hline NPI 2/12: hallucinations & $0.67(2.0)$ & $0.78(2.7)$ & 0.906 & - & - & - \\
\hline NPI 3/12: agitation/aggressivity & $5.0(2.4)$ & $1.9(3.5)$ & 0.018 & - & - & - \\
\hline NPI 4/12: depression & $4.1(3.9)$ & $2.2(2.1)$ & 0.046 & - & - & - \\
\hline NPI 5/12: anxiety & $7.0(3.8)$ & $5.7(3.6)$ & 0.332 & - & - & - \\
\hline NPI 6/12: euphoria & $2.0(3.2)$ & $0.84(2.5)$ & 0.262 & - & - & - \\
\hline NPI 7/12: apathy & $3.3(4.9)$ & $2.7(4.3)$ & 0.736 & - & - & - \\
\hline NPI 8/12: disinhibition & $3.0(2.7)$ & $0.53(1.9)$ & 0.003 & - & - & - \\
\hline NPI 9/12: irritability & $6.3(3.9)$ & $3.5(3.3)$ & 0.033 & - & - & - \\
\hline NPI 10/12: aberrant motor behaviour & $2.4(4.2)$ & $0.87(2.3)$ & 0.152 & - & - & - \\
\hline NPI 11/12: sleep disorders & $5.3(4.4)$ & $4.6(4.5)$ & 0.694 & - & - & - \\
\hline NPI 12/12: eating disorders & $3.3(4.5)$ & $2.2(3.5)$ & 0.447 & - & - & - \\
\hline
\end{tabular}

behavioural scores (MMSE scores at T1 minus T0 $(\Delta)=+1$ / +2.6 ; FAB $\Delta=+7 /+4.2$; NPI $\Delta=-5 /-18$, respectively), while non-remitters had overall stable/worse cognitive performance.

\section{Predictors of DDS}

Logistic regression analysis revealed that positive family history of PD (OR 5.105, 95\% CI 1.45 to 17.97 ; $\mathrm{p}=0.011$ ), history of premorbid depression (OR 3.2, 95\% CI 0.98 to 10.55 ; $\mathrm{p}=0.048)$, a great change in UPDRS scores between 'Off' versus 'On' state (OR 1.12, 95\% CI 1.042 to $1.207 ; \mathrm{p}=0.002$ ) and high scores in the NPI disinhibition sub-item (OR 2.04, $95 \%$ CI 1.106 to $3.776 ; \mathrm{p}=0.023$ ) were independent predictors of DDS. These covariates accounted for a large degree of model variance according to the Hosmer-Lemeshow test (goodness-of-fit statistics $=5.467 ; \mathrm{df} 8 ; \mathrm{p}=0.707$ ).

\section{DDS therapeutic management and outcome}

Among DDS, we compared clinical features and management strategies between those with resolution of addictive behaviours $(n=14 / 35,40 \%)$ versus those with unsuccessful outcome (table 3). Logistic regression analysis performed to identify predictors of DDS remission was not performed because of the small size of the two subgroups.

PD treatment optimisation

At last follow-up visit, the whole DDS sample showed a trend towards mean LEDD reduction compared to baseline (29.9\%, range $0-84.6, p=0.088$ ). Those cases with successful DDS outcome had a mean DRT reduction of more than $55 \%$ (range $31-84 \%)$ compared to baseline $(p=0.01)$, whereas mean LEDD was reduced only by $14.4 \%$ (range $0-44 \%$ ) in those whose DDS did not remit $(p=0.75)$. Optimisation of oral DRT (including association of monoamine oxidase B (MAO-B) or catechol-omethyl-transferase (COMT) inhibitors, switch from entacapone to tolcapone, extreme fractioning of daily levodopa) led to resolution of DDS only in $3 / 35$ patients $(8.6 \%)$. As expected, DRT reduction in DDS remitters was associated with improvement in dyskinesias-related UPDRS IV scores, although motor examination (according to UPDRS III scores) did not differ between the two DDS subgroups (table 3).

\section{Advanced-stage treatment}

Seventeen DDS patients underwent at least one advanced-stage treatment: CAI $(n=9,62.3 \pm 15.4 \mathrm{mg} /$ day $)$, STN-DBS $(n=7)$, DLI $(n=5,1215 \pm 348 \mathrm{mg} /$ day). These management options were not overall associated with DDS remission $(p=0.49)$; nevertheless, different strategies seemed to have different outcomes: (a) all patients on CAI persisted in their compulsive seeking of apomorphine boli and/or extra levodopa doses; (b) effective STN-DBS led to remission only in 4/7 cases; (c) DLI successfully relieved DDS in $4 / 5$ patients (one patient relapsed after divorce and was lost at follow-up). Four DDS patients received two advanced-stage therapies because of ineffectiveness in reducing addictive behaviours and subsequent motor and 
Table 3 Differential features of PD patients with DDS according to clinical outcome

\begin{tabular}{|c|c|c|c|c|}
\hline DDS & Total $(n=35)$ & Remission ( $n=14)$ & Persisting $(n=21)$ & $\mathrm{p}$ Values \\
\hline Disease duration at DDS onset, years & $10.9(3.5)$ & $10.3(4.0)$ & $11.4(3.0)$ & 0.4 \\
\hline Patient Follow-Up, years & $3.2(2.1)$ & $3.8(2.3)$ & $3.5(3.1)$ & 0.77 \\
\hline Impulse Control Disorders (n (\%)) & $23(66)$ & $12(86)$ & $11(52)$ & 0.07 \\
\hline Subjective feeling the 'high' (n (\%)) & $8(23)$ & $2(14)$ & $6(28)$ & 0.68 \\
\hline Education & $10.6(5.6)$ & $10.8(6.8)$ & $9.9(4.6)$ & 0.67 \\
\hline Poor caregiving (n (\%)) & $14(40)$ & $2(14)$ & $12(57)$ & 0.016 \\
\hline LEDD at last F-U (mean mg/day (SD)) & $1631(1564)$ & $1061(574)$ & $2344(2090)$ & 0.031 \\
\hline LEDD reduction at last F-U (mean mg/day (SD), \%) & $639.8(1008)$ & 1271 (1344), 55.4\% & $245.4(281), 14.4 \%$ & 0.02 \\
\hline$\triangle$ UPDRS part III 'Off'—'On' & $23.4(6.7)$ & $21.7(7.1)$ & $25.2(5.9)$ & 0.14 \\
\hline UPDRS part IV—Dyskinesias (items 32-to-35) & $3.5(1.7)$ & $2.2(1.0)$ & $4.3(1.6)$ & $<0.01$ \\
\hline \multicolumn{5}{|l|}{ DDS pattern } \\
\hline Compulsive Levodopa intake (n (\%)) & $32(91.4)$ & 12 & 20 & 0.72 \\
\hline Compulsive DA agonist intake (n (\%)) & $3(8.6)$ & 2 & 1 & N.S. \\
\hline Apomorphine s.c. rescue boli (n/day) & 6 & 1 & 5 & 0.38 \\
\hline \multicolumn{5}{|l|}{ DDS management } \\
\hline \multicolumn{5}{|l|}{ Optimisation of DRT } \\
\hline Add MAO-B inhibitors (n) & 4 & 2 & 2 & 0.62 \\
\hline Add COMT inhibitor (n) & 4 & 2 & 2 & 0.62 \\
\hline Entacapone switched to Tolcapone ( $\mathrm{n}$ ) & 8 & 3 & 5 & 0.98 \\
\hline Advanced stage treatment $(\mathrm{n}(\%))$ & $17(49)$ & 7 & 10 & 0.20 \\
\hline CAI/STN-DBS/DLI (n) & $8 / 8 / 5^{*}$ & $0 / 3 / 4$ & $8 / 5 / 1$ & N.S. \\
\hline DLI (\% of treatments) & $24 \%$ & $57 \%$ & $10 \%$ & 0.06 \\
\hline Antipsychotic therapy (n (\%)) & $23(66)$ & $10(71)$ & $13(62)$ & 0.72 \\
\hline Quetiapine/Clozapine/Others ${ }^{\dagger}$ & $12 / 8 / 3$ & $4 / 6 / 0$ & $8 / 2 / 3$ & N.S. \\
\hline Clozapine (total \%) & $33 \%$ & $60 \%$ & $15 \%$ & 0.04 \\
\hline Antidepressants (n (\%)) & $11(31)$ & $5(36)$ & $6(29)$ & 0.71 \\
\hline SSRI/SNRI/TCA & $8 / 3 / 0$ & $4 / 1 / 0$ & $5 / 2 / 0$ & N.S. \\
\hline Mood stabilisers ( $(\%))$ & $2(6)$ & $0(0)$ & $2(9)$ & 0.52 \\
\hline Psychological interventions ( $(\%))$ & $6(17)$ & $3(21)$ & $3(14)$ & 0.65 \\
\hline
\end{tabular}

non-motor fluctuations: CAI first followed by DLI $(n=3)$ or STN-DBS $(n=1)$. When considering the DDS patients who underwent any advanced-stage treatment $(n=17 / 35)$, levodopa infusion was the one more frequently associated with successful DDS outcome, though not reaching statistical significance $(4 / 7$ of remitters $(57 \%)$ versus $1 / 10(10 \%)$ of non-remitters, $\mathrm{p}=0.06)$. LEDD was reduced by $53.5 \pm 24.8 \%$ after STN-DBS (by 71\% (range 50.8-84.6) in those whose DDS remitted compared to $35 \%$ (range 28-32) in those who did not) and by $23.5 \pm 9.2 \%$ (range $0-25$ ) after initiation of CAI.

\section{Psychiatric treatment}

Delusions and aggressive behaviours were controlled better with clozapine than with quetiapine; olanzapine, risperidone, and even haloperidol had to be used in a few more severe cases. Clozapine was more commonly used in those whose DDS symptoms remitted $(p=0.04)$ and this was not associated with psychosis reduction $(p=0.31)$. As was to be expected in view of their worse depressive symptoms, antidepressants were more common in DDS patients, the most commonly prescribed being selective serotonin re-uptake inhibitors (SSRI) ( $\mathrm{n}=8 / 35$ cases, $22.8 \%$ ), then serotonin-norepinephrine reuptake inhibitors (SNRI) $(n=3 / 35,8.6 \%)$, tricyclics were never prescribed to the DDS cohort. Antidepressants, psychological support and mood stabilisers ( $\mathrm{n}=1$ on carbamazepine $900 \mathrm{mg} /$ day; $\mathrm{n}=1$ on lamotrigine $300 \mathrm{mg} /$ day) were not effective in reducing the addictive behaviours, despite improvement in the mood disorder. No suicidal attempt was recorded. Notably, the glutamatergic NMethyl-D-Aspartate (NMDA) antagonist memantine led to levodopa craving remission at $20 \mathrm{mg}$-daily in a 58 -year-old nondemented female patient with severe non-motor fluctuations allowing DRT reduction; discontinuation at 2-year follow-up led to symptoms relapse in 2-weeks, and so it was reintroduced, with good response and final DDS resolution.

\section{Non-pharmacological features}

Once DDS was diagnosed, caregivers and other family members were strongly encouraged to look after patients' compliance to the prescribed regimen or deliver medications themselves and carefully check for any possible hidden drugs or additional providers. Effective monitoring of drug compliance by family/caregiver was the most important non-pharmacological factor associated with long-lasting DDS remission $(p=0.001)$. Twelve of those 14 DDS subjects whose supervision was poor did not remit, even years after the onset (mean DDS follow-up $2.7 \pm 0.9$ years). Poor caregiving was associated with unmarried status $(n=3)$, caregiver parted/divorced $(n=4)$ or dead $(n=1)$, caregiver at work during the day $(\geq 8$ h-daily, $n=4)$, or 
caregiving by a parent with advanced PD $(\mathrm{n}=1)$ or with psychosis $(n=1)$. Worse performance in the recall task of the Rey auditory verbal learning test was associated with persisting DDS $(\mathrm{p}=0.019)$; although this finding may suggest specific impairment in memory functions (ie, medial temporal lobe), it could also reflect difficulties in recall strategies (ie, frontal lobe).

\section{DISCUSSION}

In the present study, our objective was to describe the features associated with compulsive medication intake at the time of the first diagnosis to help clinicians in the early recognition of at-risk patients. However, our main objective was to detail the therapeutic strategies associated with long-lasting resolution of an addictive disorder that is known to be associated with a high risk of relapse ${ }^{6}$ in the attempt to draw a roadmap for clinical management. Indeed, while risk factors for DDS have been previously investigated, ${ }^{12}$ to our knowledge, this is the first longitudinal study reporting the outcome of a 3-year follow-up of a large sample of PD patients with full-blown DDS, consecutively and systematically assessed at a tertiary referral centre.

\section{Risk factors for DDS}

General features

Our results confirm that patients with DDS are about 10 years younger at motor symptoms onset compared to control PD population, and are more likely to have a personal history of depressive symptoms and a personal and/or family history of drug abuse. ${ }^{24}$ An interesting novel finding is the positive family history for PD in first-degree relatives more frequently found in DDS than age-matched controls, a feature independently predicting DDS in our regression model. Furthermore, we did not find any difference in male gender prevalence ${ }^{24}$ or premorbid artistic profession ${ }^{25}$ between DDS cases and agematched controls. Taken as a whole, our results further support the hypothesis of pre-morbid inherited factors predisposing to DDS. Although the addictive pattern of medication abuse was focused on levodopa (up to $6 \mathrm{~g} /$ day) in the majority of patients, dopamine agonists became much more salient than levodopa in 9\% of cases, with some patients reaching up to $5 \mathrm{~g}$ LEDD (including one patient addicted to cabergoline) for weeks or even months before emergency psychiatric admission. Such an increase in salience to long-acting medications challenges the pathophysiological theories suggesting that fast-acting 'booster' plays a key role and deserves further studies. Notably, poor caregiving was strongly associated with poor DDS outcome and was one of the most common triggers for relapses. The importance of involvement of family members, as well as the general practitioner and the local pharmacist, has been previously highlighted in DDS, ${ }^{1}$ and unmarried status with subsequent poor caregiving has been also associated with ICDs ${ }^{26}$ supporting its fundamental role in long-term outcome of addictive disorders.

\section{Motor features}

The analysis of motor symptoms revealed greater change in motor performance between 'Off' and 'On' condition in DDS compared to both PD control groups. This change robustly predicted DDS at regression analysis and was not associated with better 'On' condition, but rather with worse motor performance during 'Off'. This is consistent with a previous study on DDS during acute levodopa challenge. ${ }^{27}$ This might suggest a disease-related factor predisposing to DDS, possibly due to more pronounced degeneration of the nigrostriatal motor pathway. Nonetheless, these subjects did not show any difference in scores related to 'Off' in the activities of daily living
(UPDRS II) and in daily 'Off'-related complications (UPDRS IV). These seemingly contrasting findings might be explained by the abnormal DRT abuse aim at preventing any 'Off' during the day leading to an almost steady 'On' status associated with disabling dyskinesias.

\section{Psychiatric features and treatment}

As expected, such an abnormal dopaminergic overstimulation was invariably associated with psychiatric symptoms, such as mania, psychosis or ICDs. Drug-induced psychosis in DDS had a pattern characterised by increased delusional ideation (mostly paranoia) rather than hallucinations, possibly related to the young age of subjects and the relatively preserved cognitive status, in line with reports in cocaine-abusers. ${ }^{6}$ Psychosis and aggressive behaviours were more effectively managed using clozapine than quetiapine, while 'less atypical' antipsychotics (eg, olanzapine, risperidone) and even 'typical' antipsychotics (eg, haloperidol) had to be used in some of the most severe cases requiring psychiatric admission. Two-thirds of DDS patients also had ICDs, as was to be expected in view of the shared risk factors and the relationship with high drug daily dosage. Several sources for medication may be used, also including different pharmacies and other PD patients living nearby. Prescriptions are often obtained from different providers, especially when their supply of medication is deliberately restricted by the neurologist. This is the background that leads patients to consult different specialists to find someone fulfilling their continuous requests to modify their treatment regimen and increase their daily dosage: this is called 'doctor shopping, ${ }^{8}$ and it is a feature consistently associated with less effective family/caregiver control.

\section{Cognitive profile}

We did not find any remarkable difference in the cognitive profile of DDS patients compared to matched control subjects. A relative imbalance between enhanced appetitive drive and reduced top-down inhibitory control from prefrontal cortical areas has been hypothesised to underlie the pathophysiology of ICDs in PD. ${ }^{28}$ Several authors have investigated the cognitive profile of PD patients with ICDs with controversial findings about the role of executive dysfunction. ${ }^{29}$ This first extensive assessment of cognitive functions in patients with DDS does not support a pivotal role of frontal-lobe dysfunction among disease-related predisposing factors. Some frontal functions, such as tasks involving the adaptation of behaviour according to changes in stimulus-reward contingencies, are adversely affected by dopaminergic medications, ${ }^{30}{ }^{31}$ so that the poor performance in the 'attentive matrices' task (reflecting difficulties in selective and sustained attention) and behavioural disinhibition (as reported by the caregivers at the NPI) that we found in DDS patients are very likely to be due to the higher dopaminergic medications intake. In consistence, the anecdotal findings of improved cognitive functions at NPS follow-up in DDS cases after substantial DRT reduction suggest that some frontal-lobe abnormalities might just be medication-induced, and thus, potentially reversible. ${ }^{31}$ We acknowledge that the retrospective nature of the study did not allow us to assess specific abilities (such as response inhibition, reward learning and decision making) that could best differentiate PD patients with addictive disorders from control subjects. Further prospective studies including more specific assessment of the abilities of the frontal lobe are needed to highlight cognitive dysfunction predisposing to DDS. 
DDS management and follow-up

Response to PD treatment

As outlined in all reports about DDS so far, once PD patients develop this disorder their long-term management becomes very difficult. ${ }^{1} 28{ }^{24}$ The first steps of clinical management included: optimisation of oral DRT using prolonged-release dopamine agonists; MAO-B or COMT inhibitors to provide more continuous dopaminergic stimulation, and thus, reduce overall daily dosage and 'booster' medications; the association of antipsychotics to increase patient compliance by reducing mania and delusional thoughts. Optimisation of oral DRT was associated with DDS resolution in less than $10 \%$ of cases. Overall reduction in long-acting medications (such as dopamine (DA) agonists) usually improved ICDs; nonetheless, it could be tolerated only by those patients undergoing DLI or STN-DBS because DA agonists reduction could worsen non-motor fluctuations (including dysphoria), and thus, increase the risk of relapse.

\section{Response to psychiatric treatment}

Although depressive symptoms are a definite risk factor for DDS, antidepressant medication did not eradicate addictive behaviours even in those with major mood improvements. ${ }^{32}$ Similarly, mood stabilisers improved mood swings and mania but were of no use in treating craving behaviours. Noteworthy is that memantine was associated with stable resolution of levodopa craving in a patient with severe non-motor 'Off'. Neuroadaptations in cortico-striatal glutamatergic projections are known to occur with repeated exposure to salient stimuli and to enhance motivation for reward-seeking and craving that occurs in addicted subjects, ${ }^{33}$ supporting a possible therapeutic role of NMDA antagonists in PD-related addictive disorders. Prospective controlled studies assessing safety and efficacy of memantine and anti-craving drugs (ie, opioid antagonists ${ }^{34}$ ) in PD patients with severe addictive disorders are needed.

\section{Response to advanced-stage treatment}

Almost 50\% of DDS cases underwent at least one advancedstage treatment during the study period. In contrast with previous reports, ${ }^{1}$ all patients who underwent CAI persisted in their compulsive drug seeking (mainly focused on levodopa, because rescue apomorphine boli were not permitted). On the other hand, DLI and STN-DBS were associated with long-lasting resolution of DDS in $80 \%$ and $57 \%$ of cases, respectively. This discrepancy might be explained by the differential DRT reduction in our cohort. Levodopa was completely discontinued only during DLI, while it was halved after STN-DBS but could not be discontinued during CAI. Apomorphine infusion was not used as monotherapy in our Institute because motor improvement could be achieved only using high daily dosage, which is usually not tolerated. ${ }^{35}$ In these patients, intermittent dopaminergic drug therapy (ie, oral levodopa) remained an important part of the treatment, possibly explaining the persistence of the addictive pattern of medication intake. According to recent literature, DDS seem to resolve after effective STN-DBS in close association with the extent of postoperative DRT reduction, ${ }^{36-39}$ showing that addictive behaviours did not improve or even exacerbate all patients who stayed on extremely high medication doses after surgery. In line with these studies, we found that four DDS subjects out of seven who underwent STN-DBS showed long-lasting symptoms resolution, while no case of either postoperative worsening or significant DA agonist withdrawal symptoms was recorded. Notably, we found a larger DRT reduction in those whose DDS remitted secondary to an optimised stimulation-induced control of On-Off fluctuations. As the sample size of the different procedures was too small to ascertain the differential effectiveness of each therapeutic option in the management of DDS, the present findings need to be confirmed in randomised controlled trials.

\section{CONCLUSION}

DDS is an uncommon albeit highly distressing psychiatric complication occurring in a minority of patients with advanced PD, whose susceptibility seems mainly driven by inherited factors. Clozapine, STN-DBS or DLI are often mandatory to compensate severe On-Off fluctuations and craving symptoms; antidepressants and/or mood stabilisers may be helpful but are unlikely to lead to remission of addictive behaviours. Effective supervision in every-day life seems to be the most important factor associated with long-lasting DDS resolution: it is thus highly recommended not only as effective treatment, but also among preventive strategies in high-risk individuals. We believe that DDS management should include pharmacological and non-pharmacological interventions targeted not only to patients, but also to caregivers and the whole family entourage.

Acknowledgements The authors are grateful to Vincenza Ragone, Biomedical Engineer, for statistical assistance and to the medical writer Jennifer $S$ Hartwig for assistance editing the manuscript. CS, DDG and CR would like to thank the 'Fondazione Grigioni per il Morbo di Parkinson', Milan, Italy for financial support.

Contributors RC contributed to the conception and design of the article, interpretation of the data, drafting the article and revising it critically for important intellectual content, and contributed to final approval of the version to be published. CS contributed to revising the article critically for important intellectual content and contributed to final approval of the version to be published. The other authors ( $M C$, ALZ, FN, ST, NM, CBM, GS, MZ; GP) contributed to providing clinical data and revising the article critically for important intellectual content.

Competing interests $\mathrm{RC}$ has received honoraria for symposia from Boehringer Ingelheim, Lundbeck, UCB pharma. CS, DDG and CR have received salary from the 'Fondazione Grigioni per il Morbo di Parkinson', Milan, Italy.

Ethics approval Istituti Clinici di Perfezionamento Ethics Committee.

Provenance and peer review Not commissioned; internally peer reviewed.

\section{REFERENCES}

1 Giovannoni G, O'Sullivan JD, Turner K, et al. Hedonistic homeostatic dysregulation in patients with Parkinson's disease on dopamine replacement therapies. J Neurol Neurosurg Psychiatry 2000;68:423-8.

2 Evans $A H$, Lawrence $A D$, Potts J, et al. Factors influencing susceptibility to compulsive dopaminergic drug use in Parkinson disease. Neurology 2005;65:1570-4.

3 Priebe S. Levodopa dependence: a case report. Pharmacopsychiatry 1984;17:109-10.

4 Nausieda PA. Sinemet "abusers". Clin Neuropharmacol 1985:8:318-27.

5 Witjas T, Kaphan E, Azulay JP, et al. Nonmotor fluctuations in Parkinson's disease: frequent and disabling. Neurology 2002;59:408-13.

6 Lawrence AD, Evans AH, Lees AJ. Compulsive use of dopamine replacement therapy in Parkinson's disease: reward systems gone awry? Lancet Neurol 2003;2:595-604.

7 Evans $\mathrm{AH}$, Lees AJ. Dopamine dysregulation syndrome in Parkinson's disease. Curr Opin Neurol 2004;17:393-8.

8 Katzenschlager R. Dopaminergic dysregulation syndrome in Parkinson's disease. J Neurol Sci 2011;310:271-5

9 Hughes AJ, Ben-Shlomo Y, Daniel SE, et al. What features improve the accuracy of clinical diagnosis in Parkinson's disease: a clinicopathologic study. Neurology 1992:2:1142-6.

10 Arabia G, Zappia M, Bosco D, et al. Body weight, levodopa pharmacokinetics and dyskinesia in Parkinson's disease. Neurol Sci 2002;23(Suppl 2):S53-4.

11 Siri C, Cilia R, De Gaspari D, et al. Cognitive status of patients with Parkinson's disease and pathological gambling. J Neurol 2010;257:247-52.

12 Folstein MF, Folstein SE, McHugh PR. 'Mini-mental state'. A practical method for grading the cognitive state of patients for the clinician. J Psychiatr Res 1975:12:189-98.

13 Dubois B, Slaechevsky A, Litvan I, et al. The FAB: a frontal assessment battery at bedside. Neurology 2000;55:1621-6. 
14 Heaton RK, Pendleton MG. Use of neuropsychological tests to predict adult patients' everyday functioning. J Consult Clin Psychol 1981;49:807-21.

15 Basso A, Capitani E, Laiacona M. Raven's coloured progressive matrices: normative values on 305 adult normal controls. Funct Neurol 1987;2:189-94.

16 Spinnler H, Tognoni G. Standardizzazione e taratura italiana di test neuropsicologici. Italian J Neurol Sci 1987;6(Suppl 8):47-50.

17 De Renzi E, Nichelli P. Verbal and non verbal short term memory impairment following hemispheric damage. Cortex 1975;11:33-41.

18 Wechsler D. Wechsler adult intelligence scale. New York: The Psychological Corporation, 1981.

19 Carlesimo GA, Caltagirone C, Gainotti G. The mental deterioration battery: normative data, diagnostic reliability and qualitative analyses of cognitive impairment. Eur Neurol 1996;36:378-84.

20 Yesavage JA, Brink TL, Rose $T L$, et al. Development and validation of a geriatric depression screening scale: a preliminary report. J Psychiatr Res 1983;17:37-49.

21 Cummings JL, Mega M, Gray K, et al. The neuropsychiatric inventory: comprehensive assessment of psychopathology in dementia. Neurology 1994;44:2308-14.

22 Grosset KA, Grosset DG. Proposed dose equivalence for rapid switching between dopamine agonists in Parkinson's disease. Clin Ther 2006;28:1063-4.

23 LeWitt PA, Boroojerdi B, MacMahon D, et al. Overnight switch from oral dopaminergic agonists to transdermal rotigotine patch in subjects with Parkinson disease. Clin Neuropharmacol 2007;30:256-65.

24 O'Sullivan SS, Evans AH, Lees AJ. Dopamine dysregulation syndrome: an overview of its epidemiology, mechanisms and management. CNS Drugs 2009;23:157-70.

25 Schwingenschuh $\mathrm{P}$, Katschnig $\mathrm{P}$, Saurugg $\mathrm{R}$, et al. Artistic profession: a potential risk factor for dopamine dysregulation syndrome in Parkinson's disease? Mov Disord 2010;25:493-6.

26 Weintraub D, Koester J, Potenza MN, et al. Impulse control disorders in Parkinson disease: a cross-sectional study of 3090 patients. Arch Neurol 2010;67:589-95.

27 Evans $A H$, Lawrence AD, Cresswell SA, et al. Compulsive use of dopaminergic drug therapy in Parkinson's disease: reward and anti-reward. Mov Disord 2010;25:867-76.
28 Cilia R, van Eimeren T. Impulse control disorders in Parkinson's disease: seeking a roadmap toward a better understanding. Brain Struct Funct 2011;216:289-99.

29 Poletti M, Bonuccelli U. Impulse control disorders in Parkinson' disease: the role of personality and cognitive status. J Neurol 2012;259:2269-77.

30 Swainson R, Rogers RD, Sahakian BJ, et al. Probabilistic learning and reversal deficits in patients with Parkinson's disease or frontal or temporal lobe lesions: possible adverse effects of dopaminergic medication. Neuropsychologia 2000:38:596-612.

31 Cools R. Dopaminergic modulation of cognitive function-implications for L-DOPA treatment in Parkinson's disease. Neurosci Biobehav Rev 2006:30:1-23.

32 Courty E, Durif F, Zenut M, et al. Psychiatric and sexual disorders induced by apomorphine in Parkinson's disease. Clin Neuropharmacol 1997:20:140-7.

33 Kalivas PW. The glutamate homeostasis hypothesis of addiction. Nat Rev Neurosci 2009:10:561-72.

34 Bosco D, Plastino M, Colica C, et al. Opioid antagonist naltrexone for the treatment of pathological gambling in Parkinson disease. Clin Neuropharmacol 2012;35:118-20

35 Antonini A, Isaias IU, Rodolfi G, et al. A 5-year prospective assessment of advanced Parkinson disease patients treated with subcutaneous apomorphine infusion or deep brain stimulation. J Neurol 2011;258:579-85

36 Lhommée $E$, Klinger $H$, Thobois $S$, et al. Subthalamic stimulation in Parkinson's disease: restoring the balance of motivated behaviours. Brain 2012;135:1463-77.

37 Lim SY, O'Sullivan SS, Kotschet K, et al. Dopamine dysregulation syndrome, impulse control disorders and punding after deep brain stimulation surgery for Parkinson's disease. J Clin Neurosci 2009:16:1148-52.

38 Moum SJ, Price CC, Limotai N, et al. Effects of STN and GPi deep brain stimulation on impulse control disorders and dopamine dysregulation syndrome. PLOS ONE 2012; 7:e29768.

39 De la Casa-Fages B, Grandas F. Dopamine dysregulation syndrome after deep brain stimulation of the subthalamic nucleus in Parkinson's disease. J Neurol Sci 2012;312:191-3 